\title{
Experimental monitoring of the self-heating properties of thermoplastic composite materials during tensile and cyclic tests
}

\author{
Laura Muller ${ }^{1, *}$, Jean-Michel Roche ${ }^{1}$, Antoine Hurmane ${ }^{1}$, Catherine Peyrac ${ }^{2}$, and Laurent Gornet ${ }^{3}$ \\ ${ }^{1}$ ONERA, The French Aerospace Lab, F-92322, Châtillon, France \\ ${ }^{2}$ CETIM, 52 avenue Félix Louat, 60300 Senlis, France \\ ${ }^{3} \mathrm{GeM}$, École Centrale de Nantes, 1 rue de la Noë, 44321 Nantes, France
}

\begin{abstract}
The sizing of industrial structures with a given material requires to understand completely its fatigue behaviour. Yet, the full estimation of the fatigue lifetime of a material requires time-consuming and expensive fatigue testing campaigns, and a huge number of samples. An alternative experimental procedure is based on the monitoring of the self-heating properties of the studied material. The aim is to correlate the fatigue limit with a change of the thermal behaviour of the material during the self-heating tests. The main advantage of these tests is that they need only a limited number of mechanical cycles, and require few samples to test. Ultimately, self-heating tests lead to an accelerated estimation of the fatigue behaviour. The purpose of the present paper is to validate this approach for a woven carbon/thermoplastic composite material.
\end{abstract}

\section{Introduction}

Over the years, composite materials are more and more used for primary structural components. This phenomenon is due to the high strength and stiffness to weight ratios of the composite materials. In these conditions, it has become very important to study their static and fatigue behaviour: indeed, structural components are designed with high safety factors, as the fatigue behaviour of the used composite material is not completely known and understood [1].

So far, the main method used in the industry to study the fatigue behavior of a given material is to build its Wöhler curve, also called $\mathrm{S} / \mathrm{N}$ curve, which provides the number of cycles to failure $(\mathrm{N})$ for each load level (S) (Fig. 1). The constitution of the Wöhler curve requires a large number of mechanical tests, including several samples per load level to avoid scattering, and until $10^{7}$ cycles for the lowest load levels, meaning several months of test for the lowest load levels. Moreover, at these levels, an asymptote can appear, meaning that under this threshold value, the material will not prematurely break because of fatigue. This asymptote is called "fatigue limit" and is used to size structural components [2] (Fig. 1).

An alternative to the fatigue tests are the selfheating tests. This methodology is also based on cyclic loads, but instead of cycling each sample until failure for several given load values, several blocks with a limited number of cycles for each block are applied on the same sample, for increasing load values (Fig. 2).

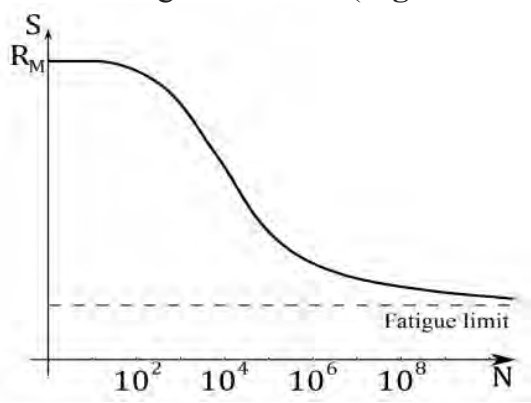

Fig. 1 : Wöhler curve and fatigue limit

During each block of fatigue cycles, the surface temperature of the sample increases until a stabilized heating value. This self-heating is due to the viscosity of the resin and for any kind of damage induced by the cyclic loading. It can be monitored by infrared camera or thermocouples, and compared block after block. The evolution of the average stabilized heating with the maximal stressed value of each block shows two tendencies: for the lowest load values, the stabilized heating is almost constant from one block to another. Then, after a particular load value, the stabilized heating strongly increases block after block. This change of the thermal behaviour may indicate an alteration of the viscous properties of the resin, and/or the initiation of fatigue damage (Fig. 3). 


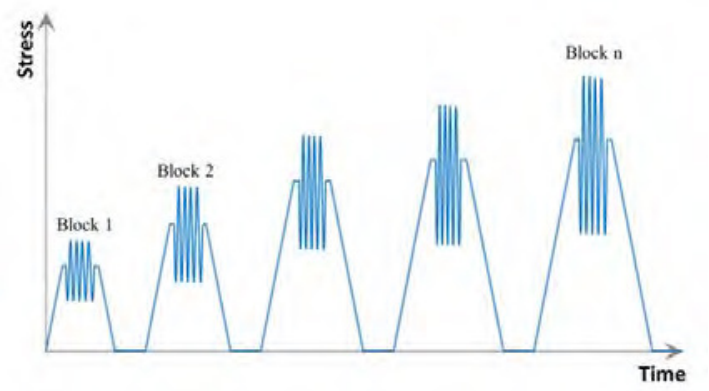

Fig. 2 : Schematic representation of self-heating tests

This approach has been proven efficient for metallic materials [3]-[7] and successfully verified for some composites [8], [9] : the specific load value between the two thermal tendencies during the self-heating tests is related to the fatigue limit determined on the Wöhler curve. It seems that the self-heating monitoring can indeed enable a reliable monitory of fatigue damage. The aim of this work is to apply this methodology to a woven carbon/thermoplastic composite material. Several experimental campaigns are carried out: a static test campaign to characterize the material's properties, conventional fatigue tests, and self-heating tests.

\section{Quasi-static tensile test campaign}

\subsection{Material and conditioning}

The studied material is a balanced plain woven carbon/thermoplastic composite. The thermoplastic matrix is a semi-crystalline polymer, the polyamide 6.6 , also called PA66. This resin has the particularity to be very sensitive to moisture absorption : the polyamid can absorb $2 \%$ to $5 \%$ moisture, depending on the relative humidity of the specimen [10], [11]. This important sensitivity to moisture has a direct impact on the mechanical properties (like the Young modulus or the ultimate tensile strength) [8], [12]. To guarantee coherence of the experimental campaigns, samples have to be conditioned with a relative humidity (RH) controlled. In this study, the samples are conditioned at ambient temperature, with a $\mathrm{RH}$ fixed at $50 \%$, to approach the ambient conditions. The conditioning is ensured by a desiccator, in which the conditioned air is generated by a saturate salted solution [13] and regularly controlled.

\subsection{Quasi-static tensile tests}

\subsubsection{Experimental procedure}

The quasi-static tensile tests are carried on a ZWICK testing machine, equipped with mechanic grips and a $150 \mathrm{kN}$ load cell. The applied load speed is fixed to 2 $\mathrm{MPa} / \mathrm{s}$. Samples are rectangular $\left(250 \times 25 \mathrm{~mm}^{2}\right)$, along three directions: $0^{\circ}, 90^{\circ}, 45^{\circ}$. The temperature field and specimen displacement are monitored respectively by an

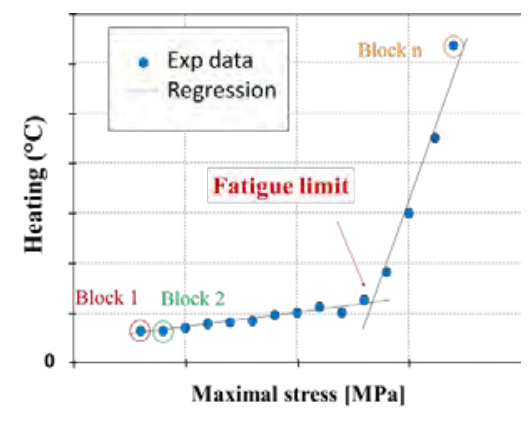

Fig. 3 : Self-heating curve

infrared camera and optical camera for Digital Image Correlation (DIC). Their characteristics are detailed in [14].

\subsubsection{Mechanical results}

Mechanical characterization involves the determination of failure stress, Shear's and Young's modulus and Poisson ratio according to the 3 orthotropic directions of the material. Only warp and weft plane properties are measured. To do this, the samples are tested up to failure, and the measured displacement fields lead to calculated strain fields. The Young modulus E is defined as the ratio between the stress $\sigma$ and the longitudinal strain $\varepsilon_{\text {longi }}$ in the elastic domain of the material. Assuming that the material strength is equivalent to a laminate composite strength, the Young modulus is experimentally determined according to the EN 2561-91 norm, i.e. by calculating the slope of $\sigma\left(\varepsilon_{\text {longi }}\right)$ on a stress range between $10 \%$ and $50 \%$ of failure stress (black curve in Fig. 4). The Poisson's ratio is defined as the ratio between the transverse $\varepsilon_{\text {transv }}$ and the longitudinal $\varepsilon_{\text {longi }}$ strain. It is determined on the slope $\varepsilon_{\text {transv }}\left(\varepsilon_{\text {longi }}\right)$ with the same interval $(10 \%$ to $50 \%$ of failure stress) as for Young's modulus.

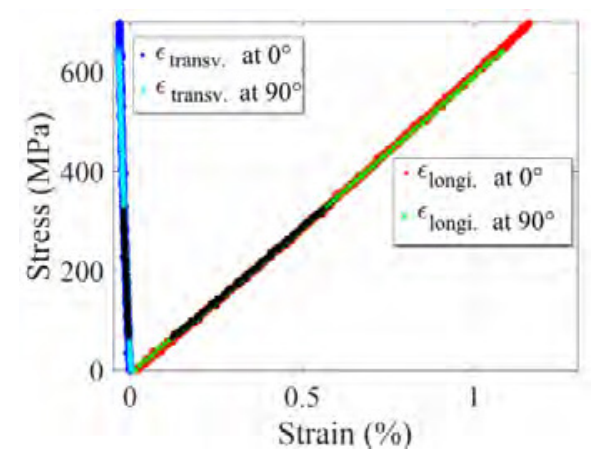

Fig. 4 : Stress-strain curve, with the longitudinal and transversal strain for sample at $0^{\circ}$ and $90^{\circ}$, and the domains used for the evaluation of the Young modulus and the Poisson's ratio (black)

Table 1 summarizes the properties of each orientation by averaging the values obtained for each sample. As shown in Fig. 4, the properties at $0^{\circ}$ and $90^{\circ}$ are similar, due to the balanced nature of the woven composite.

Corresponding author: laura.muller@onera.fr 
Table 1: Elastic properties and ultimate tensile stress of the studied carbon T700/PA66 thermoplastic composite material

\begin{tabular}{cccc}
\hline Orientation & $\begin{array}{c}\text { Ultimate tensile } \\
\text { stress }\end{array}$ & $\begin{array}{c}\text { Young } \\
\text { modulus }\end{array}$ & $\begin{array}{c}\text { Poisson's } \\
\text { ratio }\end{array}$ \\
\hline $0^{\circ}$ (warp) & $695 \mathrm{MPa}$ & $59 \mathrm{GPa}$ & 0.031 \\
$90^{\circ}$ (weft) & $595 \mathrm{MPa}$ & $58 \mathrm{GPa}$ & 0.033 \\
$45^{\circ}$ (shear) & $265 \mathrm{MPa}$ & & \\
\hline
\end{tabular}

\subsection{Thermal behaviour}

\subsubsection{Theoretical background}

Several authors [1], [15] have been studying the thermal behaviour of the material during a static tensile test. It is noted that temperature variations follow a three-step pattern:

- The first regime (I) corresponds to the elastic mechanical domain of the material, and the temperature linearly decreases with the loading stress.

- The second regime (II) corresponds to the anelastic mechanical behaviour, the temperature decreases non-linearly until a minimum.

- The third regime (III) is characterized by the nonlinear increase of the temperature, due to viscosity and damage apparition, until the sample breaks.

The linear variation of temperature $\Delta T$ in the first domain (I) is related to the stress level increase $\Delta \sigma$ by thermoelastic effect [16], [17]:

$$
\Delta T=-T_{0} K_{m} \Delta \sigma
$$

$T_{0}$ is the initial average temperature of the sample, $K_{m}$ is the thermoelastic coefficient defined by:

$$
\mathrm{K}_{\mathrm{m}}=\frac{\alpha}{\rho c_{p}}
$$

$\alpha$ is the thermal expansion coefficient, $\rho$ the mass density and $c_{p}$ the specific heat of the material. According to this law, the end of linearity of the temperature behaviour marks the end of the elastic domain. This change has been correlated with the appearance of micro-damage in [15], on thermoset composites.

Then, the transition from a drop to an increase temperature via a thermal minimum explains the transition from the second domain to the third domain. This transition was correlated with the appearance of macro damage in [15], which would generate enough heat to balance the cooling of the specimen.

This study will be interested in the threshold stress values associated with the transition from one step to another: the stress value related to the end of the elastic domain and the stress value related to the thermal minimum.

\subsubsection{Results and discussion}

In the case of a $0^{\circ}$ specimen (and a $90^{\circ}$ specimen also), the evolution of its average heating is in good agreement with the literature description, as shown in Fig. 5. To determine the two particular stress values mentioned before, the noise due to the infrared camera has to be filtered. To do this, a Gaussian filter is applied, as shown in Fig. 6. Once the thermal signal filtered, the $\ell 1$ trend filtering, related to "segmented regression", is applied : the variables (the thermal curve) are segmented into groups, and a regression analysis is performed on each segment [18], [19]. The thermal curve is interpolated as a succession of straight lines (Fig. 7), and the number of segments is defined by the $\ell 1$ trend filtering so that the correlation coefficient between the curve and the line is minimized. The accuracy of this minimization can be adjusted according to the $\ell 1$ parameter $\lambda$.

The junctions between two straight lines are highlighted by dots, Fig. 7. The two particular values that interest us are the one marking the end of the linear domain, i.e. the end of the first line; and the one corresponding with the minimal thermal value. In order to estimate a margin of error, a circular permutation of the thermal signal is applied, while maintaining the general trend of the curve: this is to redistribute the noise. Then, the $\ell 1$ trend-filtering is applied for each circular permutation step, and the new values of the junctions are saved. The process is repeated a hundred times, and the set of stored values for each junction will give us a margin of error (Fig. 8). The same procedure can is applied for a $90^{\circ}$ specimen. The particular stress values are summarized in Table 2 .

Table 2 : Particular stress values for $0^{\circ}$ et $90^{\circ}$ specimens

\begin{tabular}{ccc}
\hline Orientation & End of linearity & Thermal minimum \\
\hline $\begin{array}{c}\text { Particular stress values } \\
\text { at } 0^{\circ}\end{array}$ & $165 \mathrm{MPa}$ & $360 \mathrm{MPa}$ \\
Particular stress values & $115-215] \mathrm{MPa}$ & {$[340-380] \mathrm{MPa}$} \\
at $90^{\circ}$ & {$[187-205] \mathrm{MPa}$} & $347 \mathrm{MPa}$ \\
\end{tabular}

The next step of the work consists in connecting these particular stress values to physical phenomena, especially to damage apparition. Two acoustic sensors were used to record each acoustic emission occurring during the test. The plotting of the cumulative energy as a function of the applied stress shows a starting threshold for damage, defined by the initiation of first acoustic emissions, as shown in Fig. 9. This threshold has been determined shortly before $400 \mathrm{MPa}$ for $0^{\circ}$ specimens, corresponding to their thermal minimum. As the damage in a composite is assumed to be the same during a static or a fatigue tests, the starting threshold for damage in static might be a good approximation of the fatigue limit, which finally defines the low stress from which fatigue damage occurs. The rest of the work will consist in validating this theory by analysing the acoustic exploitation or microscopic scans, and to analyse the case at $45^{\circ}$, which present a totally different thermal behaviour. 


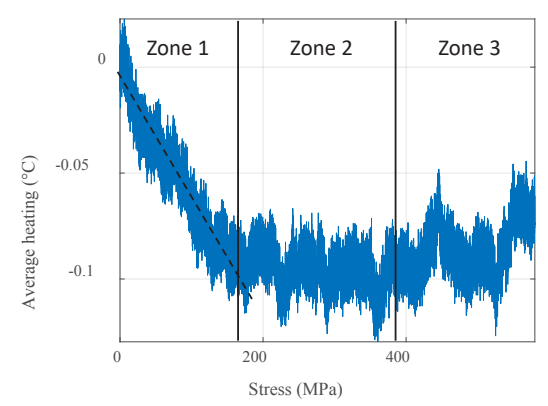

Fig. 5 : Evolution of the spatial average heating of the sample with the stress during a static test

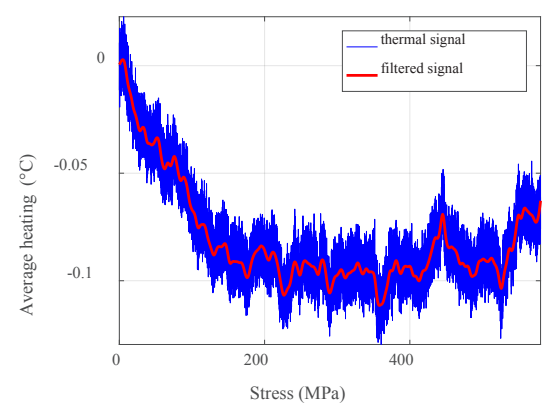

Fig. 6 : Filtering of the thermal signal

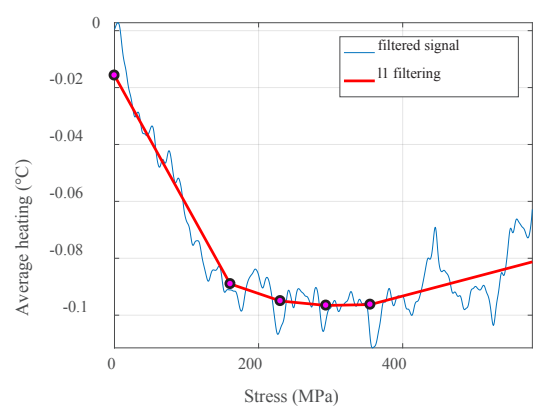

Fig. 7 : Application of $\ell 1$ trend filtering on the filtered signal

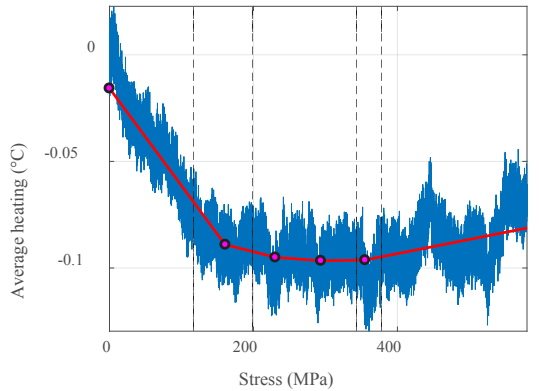

Fig. 8 : Incertitude range for the two particular stress values

\section{Fatigue tests campaigns}

Wöhler curves are traditionally used to determine the fatigue behaviour of a material, and lower stress levels will give the fatigue limit, used to size structures. The aim of the study is to test an alternative method (the selfheating monitoring approach) but a fatigue test campaign is necessary to have a reference. This campaign is carried out at CETIM. The Wöhler curves are built for the $0^{\circ}$ and the $45^{\circ}$ specimens.

\subsection{Experimental setup}

The fatigue tests are carried out on a MTS testing machine, equipped with hydraulic grips and a $25 \mathrm{kN}$ load cell. The load ratio, defined by the ratio between the minimal and the maximal stress applied, is fixed at $R=0.1$. Due to the high temperature rise of the matrix during the $45^{\circ}$ tests, the Wöhler curve at $45^{\circ}$ is realized with a cyclic load fixed at $5 \mathrm{~Hz}$, and an air-cooling system keeps the specimen at $22^{\circ} \mathrm{C}$. For the $0^{\circ}$ tests, it is the fibers that are mainly under stress, the matrix does not heat up much, which makes it possible to carry out tests at $10 \mathrm{~Hz}$ which reduces the test time.

With rectangular specimens, the break occurs at the grips outlet, due to overloads, which disqualifies the test. To solve this problem and force the failure at the center of the sample (i.e. a valid rupture), the specimens are shaped with a dogbone geometry, following the recommendation of [20]. The dimensions are then:

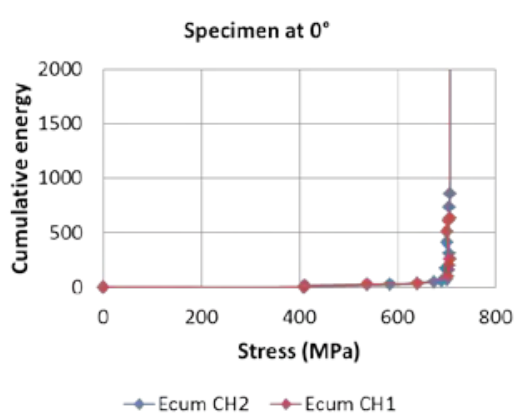

Fig. 9 : Cumulative energy measured by two acoustic sensors during a quasi-static tensile test, for a $0^{\circ}$ specimen

$20 \mathrm{~mm}$ width at the centre against $25 \mathrm{~mm}$ width in the grips, for a $200 \mathrm{~mm}$ length. About thirty specimens are used for each orientation, to build the final curves.

\subsection{Results and discussion}

The obtained Wöhler curves are presented Fig. 10 and Fig. 11. In both cases, the failure stress is recalculated from the measured thickness and the measured breaking width, in order to approach the real failure stress. To determine the fatigue limit, several models exist, listed by [21]. The Basquin model is mainly used to model the linear part, in a log-log scale. Stromeyer model include the asymptotic part in the Basquin model. Finally, the Bastenaire approximation, more complete, models the Wöhler curve from the highest to the lowest levels. Using the ESOPE software developed by Arcelor, these models are applied to the curves, the used formulas are summarized in Table 3, with A, B and C coefficients of the fit, $\mathrm{N}$ the number of cycles, $\mathrm{S}$ the maximal stress, and $\mathrm{E}$ the fatigue limit. The ESOPE software may plot the interpolation at $50 \%$ (the same probability to have an experimental dot to the left or right of the model), framed by the $10 \%$ and $90 \%$ interpolations.

Table 3 : Formulas of the main models used to fit the Wöhler curves

\begin{tabular}{c|c|c|c} 
Model & Basquin & Stromeyer & Bastenaire \\
\hline Formulas & $\log N=A \cdot s+B$ & $N=\frac{A}{s-E}$ & $\mathrm{~N}=\mathrm{A} \cdot \frac{\mathrm{e}^{-\left(\frac{s-E}{B}\right)^{C}}}{s-E}$
\end{tabular}


For the study, it is the Bastenaire model that will be applied to both curves, in order to have both the fatigue behaviour of the material and the fatigue limit (Fig. 12 and Fig. 13). The fatigue limits are determined around $500 \mathrm{MPa}$ for the $0^{\circ}$ direction and $180 \mathrm{MPa}$ for the $45^{\circ}$ direction.

For the $0^{\circ}$ specimens, the fatigue limit at $0^{\circ}$ is much higher than what could be assumed from the static tests. However, it can clearly be seen that the scattering is very important on the fatigue tests, and that the material does not seem to be subjected to fatigue damage: it has a brittle behaviour, and samples sharply break. In this case, the relevance of the use of Wöhler

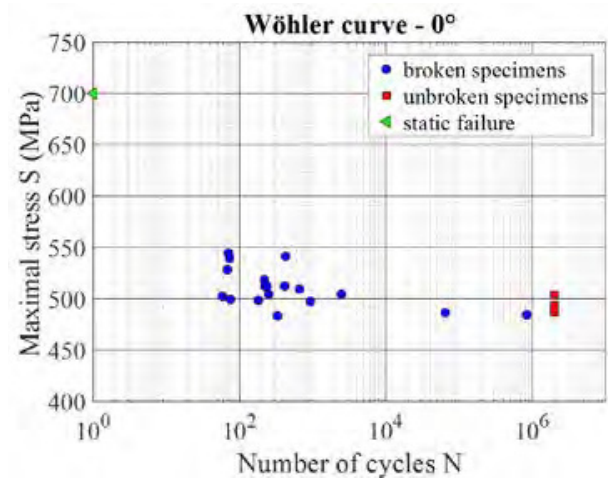

Fig. 10 : Wöhler curve at $0^{\circ}$

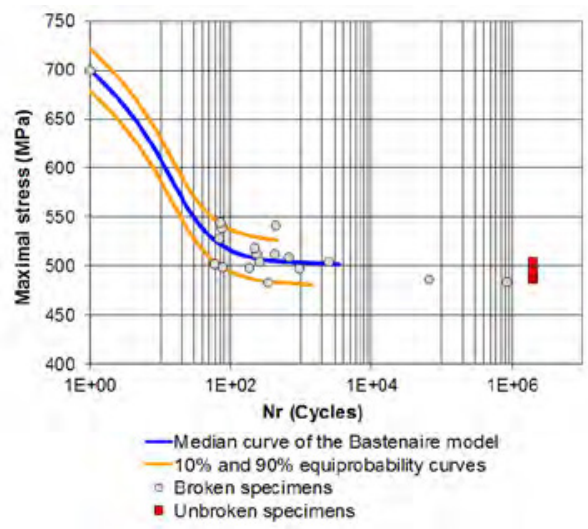

Fig. 12 : Wöhler curve at $0^{\circ}$ approximated by Bastenaire model

\section{Self-heating tests campaign}

As shown before, in the case of some composite materials, the analysis of Wöhler curves might not be concluding and satisfying to determine the fatigue limit of a material. That's why the self-heating tests are investigated, to bring another point of view of fatigue behaviour, and hopefully more robust information.

\subsection{Theoretical approach}

During a cyclic mechanical loading, characterized by its pulsation $\omega=2 \pi f$ (with $f$ the frequency of the curve can be discussed. Indeed, in the case of composites, the damage propagation (i.e. fatigue behaviour) depends on the matrix behaviour, whereas the failure of the specimen, reported on the Wöhler curve, is led by the fiber's break. It seems that two separated phenomenon (fatigue and failure), depending on two different component, are mixed on the Wöhler curves. The advantages of self-heating tests are then twofold: it would not only reduce the cost of testing (compared to conventional fatigue tests), but this new methodology might also provide greater precision on the material's fatigue behaviour.

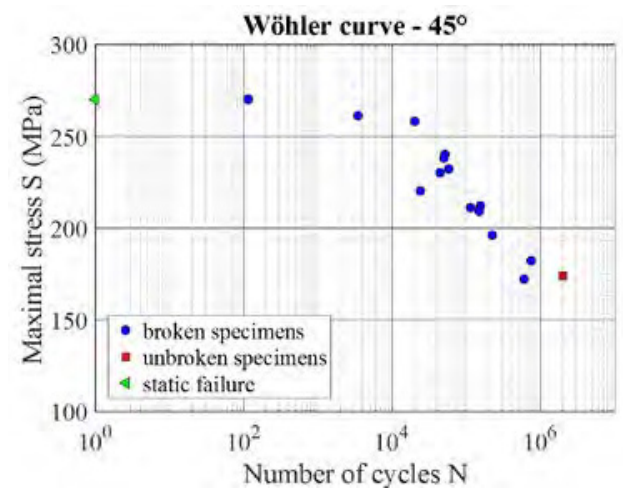

Fig. 11 : Wöhler curve at $45^{\circ}$

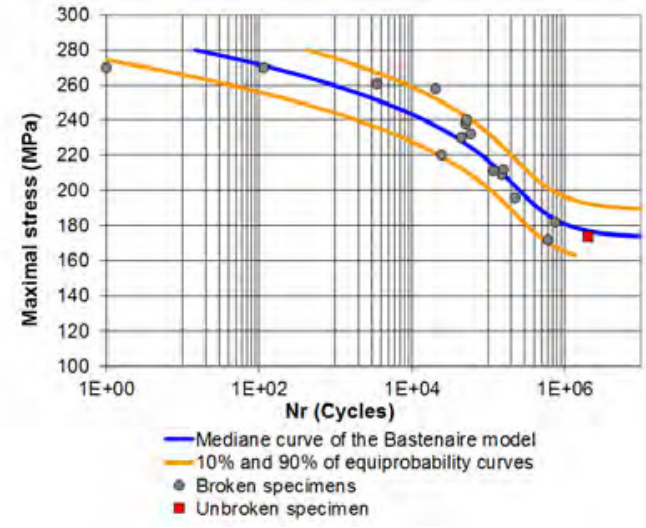

Fig. 13 : Wöhler curve at $45^{\circ}$ approximated by Bastenaire model

solicitation), the global thermal signal can be written as a Fourier decomposition:

$T(t)=T_{\text {drift }}(t)+\sum_{n=1}^{\infty} a_{n} \cos (n \omega t)+\sum_{n=1}^{\infty} b_{n} \sin (n \omega t)$

With $a_{n}$ and $b_{n}$ the Fourier coefficients, $t$ the time, $T$ the temperature, $T_{\text {drift }}$ the thermal drift of the signal.

In this study, only the first harmonic $(n=1)$ is considered. The development of (3) is detailed in [14], with the introduction of the heating $\theta(t)=T(t)-$ $T(t=0)$, of the amplitude $A_{1}$ and the phase $\phi_{1}$ of the first harmonic, of the stabilized temperature rise $\theta_{F}$ and of the characteristic time parameter $\tau$. Ultimately, the heating can be written as: 


$$
\theta(t)=\theta_{\text {drift }}(t)+A_{1} \cos \left(\omega t+\phi_{1}\right)
$$

With the first term developed as:

$$
\theta_{\text {drift }}(t)=\theta_{F}\left(1-e^{-\frac{t}{\tau}}\right)
$$

Experimentally, the thermal drift is fitted by $\theta_{\text {drift }}$, and the stabilized temperature rise $\theta_{F}$ is used as a thermal indicator for each load level during the self-heating tests. The oscillating part of the thermal signal corresponds to the first harmonic of the thermal signal, defined by its amplitude $A_{1}$ and its phase $\phi_{1}$. This harmonic describes the thermoelastic behavior of the material.

The self-heating tests are carried out on a $100 \mathrm{kN}$ hydraulic machine, equipped with hydraulic cooled grips. To avoid any resonance effect between the frequency of the test and of the infrared camera (especially for the study of the harmonic), the frequency of the cyclic load is $f=4.72 \mathrm{~Hz}$. The stress ratio is the same as for the fatigue tests, i.e. $R=0.1$. The tests are based on 12 blocks of cycles, 6,000 cycles per block. The displacement and thermal fields are respectively monitored by an optical camera dedicated to image correlation and an infrared camera (FLIR sc7000) similar to the one used for the static mechanical tests.

\subsection{Experimental passive thermal monitoring at law IR camera frequency acquisition}

The first approach, following the methodology described in the literature, is to monitor the temperature rise of the specimen with a low frequency acquisition (1 image per second), in order to analyse the thermal drift while minimizing the volume of recorded data. As shown in Fig. 14 and Fig. 15, the stabilized temperature rise value $\theta_{F}$ is reached for different stress levels, and tends to increase with the stress level. The evolution of $\theta_{F}$ with the maximal stress level on each block leads to build the "self-heating curves", displayed in Fig. 16 for three specimens: one at $0^{\circ}$ and two at $90^{\circ}$. The stake here is to

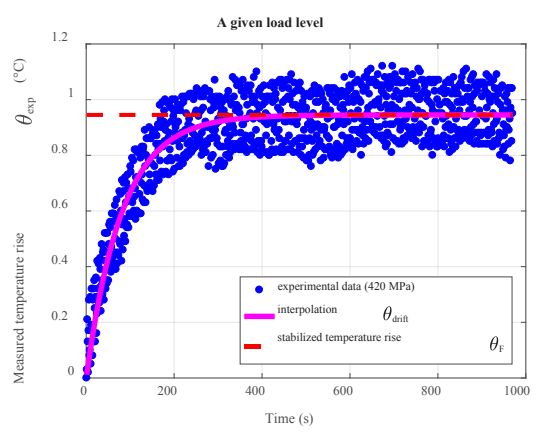

Fig. 14 : Measured self-heating $\theta_{\exp }$ for a given stress level and estimation of the stabilized temperature rise $\theta_{\mathrm{F}}$. Thermal acquisition setup: 1 image per second. Composite at $90^{\circ}$ identify a change of thermal behavior, using two linear regressions, for a threshold stress value which will be compared to the fatigue limit, in order to verify if this thermal behavior change can be directly related to fatigue damage. The method used to identify the threshold value is the similar as for tensile tests: the best intersection between two linear regressions is kept, for several redistributions of the experimental data, and the several intersection data calculated produce a confidence interval.

For both orientations $\left(0^{\circ}\right.$ and $\left.90^{\circ}\right)$, the self-heating curves show a definite variation of the thermal behavior. However, the precise and reliable identification of a threshold stress value associated with a clear change of heating tendency is not obvious. If the determined values for the $90^{\circ}$ specimens seem reliable, the lack of data to define the second domain for the $0^{\circ}$ specimen makes it impossible to properly estimate a stress threshold associated with a change of thermal behavior. Moreover, the values present a high scattering with a large confidence interval, as shown in Table 4. Despite the scattering, it would appear that the particular stress value of the self-heating curves is close to the particular stress value related to thermal minimum, on the thermal curve of quasi-static tensile tests. The exploitation of the selfheating tests has to be improved and completed with acoustic and mechanical data, to confirm this link between static tests and self-heating tests, and to better understand why the value obtained is below that the one obtained from the Wöhler curve.

Table 4 : Threshold stress values for each specimen, and their confidence range

\begin{tabular}{ccc}
\hline Specimen & $\begin{array}{c}\text { Particular stress } \\
\text { value }\end{array}$ & Confidence interval \\
\hline $\mathrm{N}^{\circ} 1$ at $90^{\circ}$ & $280 \mathrm{MPa}$ & {$\left[\begin{array}{ll}193 & 367] \\
\mathrm{MPa}\end{array}\right.$} \\
$\mathrm{N}^{\circ} 2$ at $90^{\circ}$ & $320 \mathrm{MPa}$ & {$[268347] \mathrm{MPa}$} \\
\hline
\end{tabular}

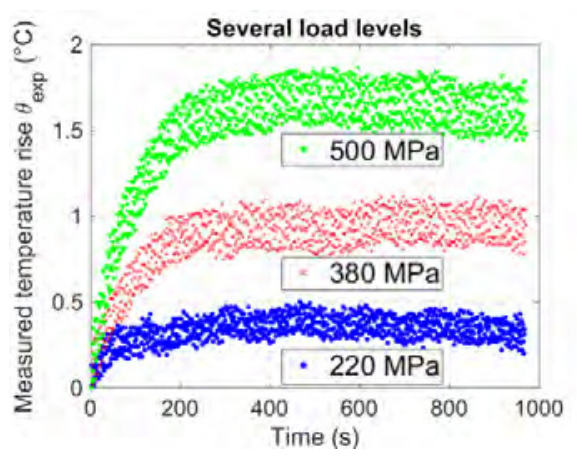

Fig. 15 : Variation of temperature rise between from one stress level to another. Composite at $90^{\circ}$ 

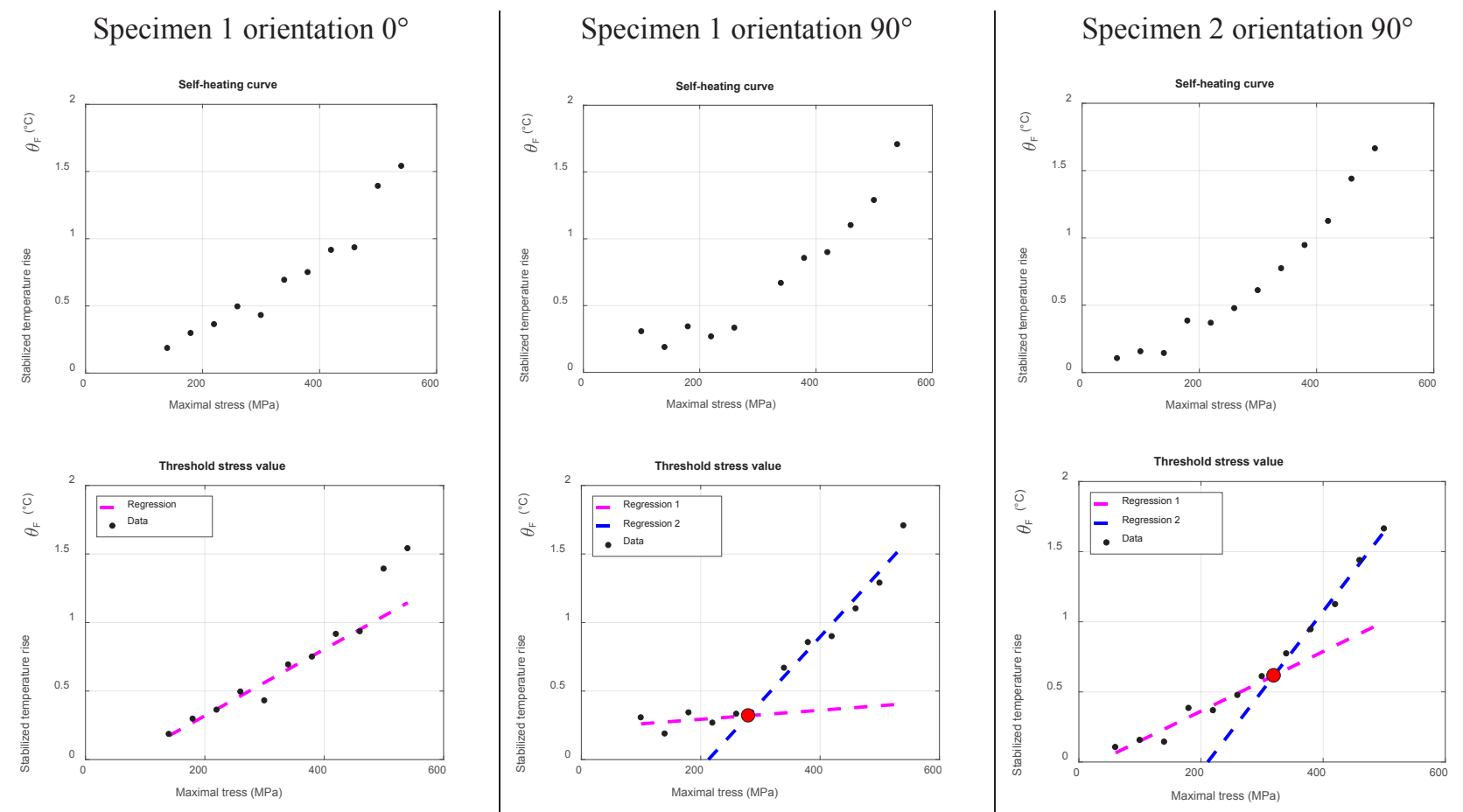

Fig. 16 : Self-heating curves and estimation of the threshold stress values

\subsection{Experimental lock-in thermal monitoring at high IR camera frequency}

The second chosen approach is to monitor the heating of the sample with a much higher frequency acquisition of 100 images per second in order to analyze the sinusoidal part of the thermal signal, in anti-phase with the mechanical signal (Fig. 17). This lock-in thermography approach had been used by Krapez et al. for metallic materials [22]. If this approach is validated, it has several advantages over the first one: the number of cycles per block may be shorten, and thus the environment has less influence on the result. Indeed, even if the thermal drift does not stabilize, is too small to be detected correctly, or is disturbed by an air current, the sinusoid component of the thermal signal will still be usable. For this study, to be able to compare results with "classic" self-heating tests, the applied mechanical load is the same as for the thermal drift study (12 blocks of cycles, 6,000 cycles per block, with the same load values). Thus the material is submittted to the same state of strain and temperature. For each block of cycles, the infrared camera is triggered to record 10 seconds (about 50 cycles), after 20 and 3,000 mechanical cycles.

Since the load ratio $\mathrm{R}$ is constant the stress amplitude grows from one block to another. According to the thermoelastic theory [16], the thermal amplitude $A_{1}$ is expected to increase proportionally as long as the material behavior remains in its elastic domain. But during the last blocks, a non-linearity of $A_{1}\left(\sigma_{\max }\right)$ is observed, using the same method of double linear regressions than for the thermal drift. This change appears at a threshold stress value (Fig. 18). Table 5 shows the threshold stress values estimated from the $A_{1}\left(\sigma_{\max }\right)$ curve, for two samples (one of each orientation). It appears that the threshold stress value is the same in both cases, with a relatively low scattering. This means that viscous phenomena and/or damage events altering the thermoelastic behaviour are activated at the same stress level. It also appears that this threshold stress value is close to the one associated with the thermal minimum during static tests, much more clearly than with the thermal drift study. So, even if the physical phenomenon of the non-linearity of the first harmonic are not yet understood (that will be the next step of this work), this approach seems at least as promising as the thermal drift study. As for the quasi static tensile tests, the case of the $45^{\circ}$ specimen will be study separately, as the thermal behavior is different than for $0^{\circ}$ and $90^{\circ}$ specimens.

Table 5 : Threshold stress value assessed from the evolution of the first harmonic amplitude, and their confidence range

\begin{tabular}{ccc}
\hline Specimen & $\begin{array}{c}\text { Threshold stress } \\
\text { value }\end{array}$ & Confidence interval \\
\hline $\mathrm{N}^{\circ} 2$ at $0^{\circ}$ & $321 \mathrm{MPa}$ & {$[290352] \mathrm{MPa}$} \\
$\mathrm{N}^{\circ} 3$ at $90^{\circ}$ & $320 \mathrm{MPa}$ & {$[306331] \mathrm{MPa}$}
\end{tabular}




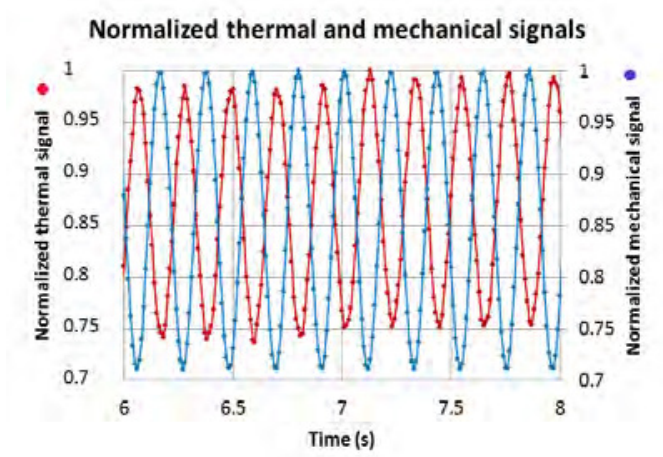

Fig. 17 : Anti-phase variations of the thermal and mechanical signals. Thermal acquisition setup: 100 images per second.

\section{Conclusion and outlooks}

After checking that the self-heating evolutions described before for thermoset composites are still valid for woven carbon fiber thermoplastic matric composite materials in [14], the aim of this paper was to develop the exploitation of the several campaigns and to bring the fatigue tests as a comparison.

It appears that the particular threshold values highlighted during the quasi static tensile tests, (especially the one associated with the thermal minimum), and during the self-heating tests (the stabilized temperature rise determined from the thermal drift, the first harmonic determined on the sinusoid component), seem to be linked. New tests, as well as new methods of exploitation, are planned in order to better understand the origin of these thermal changes, and if they might be compared.

However, the constitution of the Wöhler curves did not make it possible to link these changes in thermal behaviour to a fatigue limit. The question will be: which method is most relevant to precisely understand the fatigue phenomena of woven composite material.

\section{References}

[1] C. Colombo, F. Libonati, F.Pezzani, A. Salerno, and L. Vergani, 'Fatigue behaviour of a GFRP laminate by thermographic measurements', Procedia Eng., vol. 10, pp. 3518-3527, 2011.

[2] N. E. Dowling, Mechanical Behavior of Material : Engineering methods for deformation, fracture, and fatigue, International Edition. Pearson, 2013.

[3] M. P. Luong, 'Infrared thermographic scanning of fatigue in metals', Nucl. Eng. Des., vol. 158, no. 2-3, pp. 363-376, 1995.

[4] G. La Rosa and A. Risitano, 'Thermographic methodology for rapid determination of the fatigue limit of materials and mechanical components', Int. J. Fatigue, vol. 22, no. 1, pp. 6573, 2000.

[5] C. Doudard, S. Calloch, F. Hild, and S. Roux, 'Identification of heat source fields from infrared thermography: Determination of "self-heating" in a dual-phase steel by using a dog bone sample', Mech. Mater., vol. 42, no. 1, pp. 55-62, Jan. 2010.

[6] R. Munier, C. Doudard, S. Calloch, and B. Weber, 'Identification of the micro-plasticity mechanisms at the origin of self-heating under cyclic loading with low stress amplitude', Int. J. Fatigue, vol. 103, pp. 122-135, Oct. 2017.

[7] M. Poncelet, C. Doudard, S. Calloch, F. Hild, B. Weber, and A. Galtier, 'Prediction of self-heating measurements under

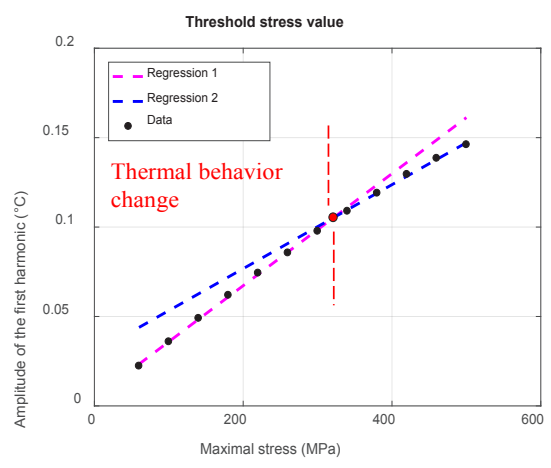

Fig. 18 : Threshold stress value of $A_{1}\left(\sigma_{\text {maxi }}\right)$

proportional and non-proportional multiaxial cyclic loadings', Comptes Rendus Mécanique, vol. 335, no. 2, pp. 81-86, Feb. 2007.

[8] L. Gornet, O. Westphal, A. Krasnobrizha, P. Rozycki, C. Peyrac, and F. Lefèbvre, 'Rapid determination of the high cycle fatigue limit of a woven carbon fibe thermoplastic matric', JNC 19, 2015.

[9] L. Jegou, Y. Marco, V. Le Saux, and S. Calloch, 'Fast prediction of the Wöhler curve from heat build-up measurements on Short Fiber Reinforced Plastic', Int. J. Fatigue, vol. 47, pp. 259-267, Feb. 2013.

[10] A. Malpot, F. Touchard, and S. Bergamo, 'Effect of relative humidity on mechanical properties of a woven thermoplastic composite for automotive application', Polym. Test., vol. 48, pp. 160-168, 2015.

[11] M. Broudin et al., 'Water diffusivity in PA66: Experimental characterization and modeling based on free volume theory', Eur. Polym. J., vol. 67, pp. 326-334, Jun. 2015.

[12] A. Benaarbia, A. Chrysochoos, and G. Robert, 'Influence of relative humidity and loading frequency on the PA6.6 cyclic thermomechanical behavior: Part I. mechanical and thermal aspects', Polym. Test., vol. 40, pp. 290-298, Dec. 2014.

[13] L. Greenspan, 'Humidity Fixed Points of Binary Saturated Aqueous Solutions', Phys. Chem., vol. 81 A, 1977.

[14] L. Muller et al., 'Experimental monitoring of the self-heating properties of thermoplastic composite materials', Procedia Eng., vol. 213, pp. 183-191, 2018.

[15] L. Vergani, C. Colombo, and F. Libonati, 'A review of thermographic techniques for damage investigation in composites', Frat. Ed Integrità Strutt., 2014.

[16] W.-T. Kim, M.-Y. Choi, Y.-H. Huh, and S.-J. Eom, 'Measurement of thermal stress and prediction of fatigue for STS using Lock-in thermography', Proc 12th-PCNDT, 2006.

[17] A. Salerno, A. Costa, and G. Fantoni, 'Calibration of the thermoelastic constants for quantitative thermoelastic stress analysis on composites', Rev. Sci. Instrum., vol. 80, no. 3, p. 034904, Mar. 2009.

[18] S.-J. Kim, K. Koh, S. Boyd, and D. Gorinevsky, '11 Trend Filtering', SIAM Rev., vol. 51, no. 2, pp. 339-360, May 2009.

[19] A. Moghtaderi, P. Borgnat, and P. Flandrin, 'Trend filtering: Empirical Mode Decompositions versus 11 and HodrickPrescott', Adv. Adapt. Data Anal., vol. 03, no. 01n02, pp. 4161, Apr. 2011.

[20] I. De Baere, W. Van Paepegem, C. Hochard, and J. Degrieck, 'On the tension-tension fatigue behaviour of a carbon reinforced thermoplastic part II: Evaluation of a dumbbellshaped specimen', Polym. Test., vol. 30, no. 6, pp. 663-672, Sep. 2011.

[21] E. Castillo and A. Fernández-Canteli, A unified statistical methodology for modeling fatigue damage. Springer Science \& Business Media, 2009.

[22] J. C. Krapez, D. Pacou, and G. Gardette, 'Lock-in thermography and fatigue limit of metals', Off. Natl. ETUDES Rech. Aerosp. ONERA-Publ.-TP, no. 187, 2000.

\footnotetext{
Corresponding author: laura.muller@,onera.fr
} 